W. Robert J. Funnell

\title{
On the undamped natural frequencies and mode shapes of a finite-element model of the cat eardrum
}

Published in:

Journal of the Acoustical Society of America, 73(5), May 1983, pp.

1657-1661

Copyright (1983) Acoustical Society of America. This article may be downloaded for personal use only. Any other use requires prior permission of the author and the Acoustical Society of America.

doi:10.1121/1.389386

http://scitation.aip.org/content/asa/journal/jasa/73/5/10.1121/1.389386 


\title{
On the undamped natural frequencies and mode shapes of a finite-element model of the cat eardrum
}

\author{
W. Robert J. Funnell \\ BioMedical Engineering Unit and Department of Otolaryngology, McGill University, 3655, rue Drummond, \\ Montréal, Québec, Canada H3G 1 Y6
}

(Received 10 Octọber 1982; accepted for publication 27 January 1983)

This paper presents a three-dimensional finite-element model of the cat eardrum which includes inertial effects. The model is implemented using a hierarchical modeling scheme which permits the mesh resolution to be varied. The static behavior of the model is calculated as a function of mesh resolution in order to check the validity of an earlier model. The first six undamped natural frequencies, and the corresponding modal vibration patterns, are then calculated. They are found to lie between about 1.8 and $3.2 \mathrm{kHz}$ for the standard values chosen for the model parameters. The effects on the natural frequencies of varying seven parameters of the model are described.

PACS numbers: 43.63.Bq, 43.63.Hx, 43.66.Gf

\section{INTRODUCTION}

In an earlier paper (Funnell and Laszlo, 1978) a threedimensional linear finite-element model of the cat eardrum was presented. In that paper only the low-frequency behavior of the model was considered; that is, the behavior in the region below about $1 \mathrm{kHz}$ where inertial effects are not important. In a later conference presentation (Funnell, 1980) the higher-frequency behavior was considered. However, the element mesh of the model presented in those two papers was quite coarse, making the higher-frequency results particularly suspect since they involved more complicated vibration patterns.

This paper presents a model which is essentially unchanged except that it has an element mesh which can be made finer than that of the earlier model. Section I discusses the definition of the model, and the way in which it can be given various mesh resolutions. Section II presents the static behavior of the model, studying the effect of mesh resolution and comparing it to the earlier model. Section III presents the undamped natural frequencies and mode shapes of the model, and Sec. IV discusses the effects of varying certain parameters of the model.

\section{MODEL DEFINITION}

\section{A. Mesh generation}

The model presented here is implemented using a hierarchical modeling scheme. This paper gives only a brief description as it applies to the current model of the cat eardrum; the details of the model-generation software will be described elsewhere.

At the lowest level of the hierarchy, the three-dimensional coordinates of named control points are specified which define the overall geometry of the model. In the model considered here there are 20 control points defining the outline of the eardrum (taken to be lying in the $x-y$ plane), 14 defining the outline of the manubrium, another four points which define the boundary between the pars tensa and the pars flaccida, and finally two points defining the position of the ossicular axis of rotation.
At the next level of the hierarchy, curved three-dimensional line segments are defined using the names of the above control points, by means of move, line, and spline commands. The move command is used to start a line segment, the line command generates a straight line segment, and the spline command produces a curved line segment using quasicubic splines (Hazony, 1979). In addition to this geometrical definition, one can also specify the boundary conditions corresponding to each line: the lines defining the outline of the drum are fully clamped (that is, all six degrees of freedom at each node are fixed); the lines describing the manubrium (assumed to be rigid) are slaved to the line defining the ossicular axis of rotation; and the lines defining the boundary between pars tensa and pars flaccida are completely free.

The third level of the hierarchy consists of definitions of surface regions, the outline of each being defined by linking together the lines defined earlier. In addition to the geometry one can define for each region (1) boundary conditions for internal nodes; (2) the material type (see Sec. ID); and (3) the thickness. In this model there are regions for the pars tensa (free, material 1, $40 \mu \mathrm{m}$ thick); the pars flaccida (free, material 3, $40 \mu \mathrm{m}$ thick); the manubrium (slaved, material $2,40 \mu \mathrm{m}$ thick); and two small regions representing the ligament forming the boundary between the pars tensa and pars flaccida (free, material $1,300 \mu \mathrm{m}$ thick).

Once the model is defined as described above, one can specify the desired mesh resolution $\boldsymbol{n}$ as a nominal number of elements across the diameter of the model. The "diameter" $d$ is estimated as the largest of $x_{\max }-x_{\min }, y_{\max }-y_{\min }$, and $z_{\max }-z_{\min }$; the computer program calculates a line-segment length of $d / n$, then subdivides each line of the model definition into equal straight-line segments of approximately that length. It then generates a two-dimensional triangular mesh over the projection of each surface region of the model onto the $x-y$ plane. The algorithm attempts to make all of the triangular elements nearly equilateral. This mesh generation is completely automatic except for a small amount of interaction required to guide the process for the coarser meshes $(n<10)$. In Fig. 1 are shown the meshes generated for four values of $n$ between 6 and 15 . 

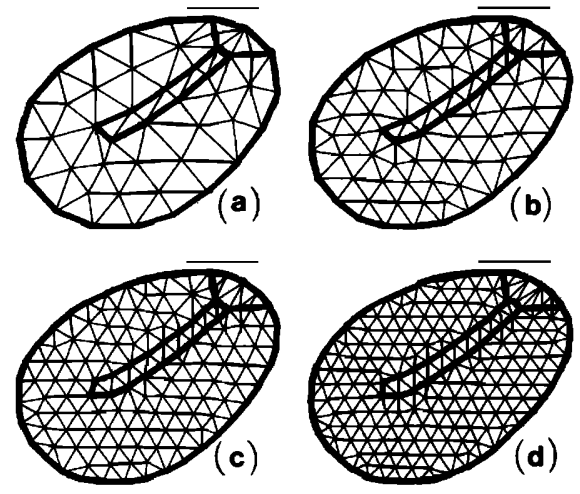

FIG. 1. Finite-element meshes generated for various values of the meshresolution parameter $n$. (a) $n=6$, (b) $n=9$, (c) $n=12$, (d) $n=15$.

\section{B. Three-dimensional curvature}

The three-dimensional curvature of the eardrum model is obtained by assigning appropriate $z$ coordinates to the internal nodes produced by the mesh-generation process. The desired curvature is specified as a normalized radius of curvature $c$, as in Funnell and Laszlo (1978). Each node within the region corresponding to the pars tensa is considered to be located on a circular arc which lies in a plane perpendicular to the plane of the tympanic ring (the $x-y$ plane), with one end on the manubrium and the other on the tympanic ring; the radius $r_{f}$ of this arc (or "fiber") is given by

$$
r_{f}=c d_{f},
$$

where $d_{f}$ is the straight-line distance between the ends of the fiber. The only difference between this representation of the curvature and that used in our earlier model lies in how the end points of each fiber are located along the manubrium and along the tympanic ring. In the earlier model the arcs were positioned first by eye, and then the nodes were forced to lie on them (see Fig. 1 in the 1978 paper). In the present model the $x-y$ position of each internal node is necessarily determined by the mesh-generation process; fiber endpoints are then calculated to correspond to the shortest fiber passing through that node. The standard value of $c$ used here is 1.19; this value was used in the 1978 paper because it was the smallest value that did not result in any nodes lying above the plane of the tympanic ring, and it is used here for consistency. The result is an overall shape very similar, but not identical, to that of the old model.

\section{Bandwidth minimization}

Once the complete finite-element model has been generated, it is processed through a bandwidth-minimization program which renumbers the nodes in order to reduce the bandwidth of the system stiffness matrix, using the algorithm of Crane et al. (1976), adapted to the SAP finite-element program used here (see Sec. IE). This minimization is important because the automatic mesh generation produces very large bandwidths. The process is both fast and effective: applied to the 1978 eardrum model, in a couple of seconds it produced a bandwidth $13 \%$ smaller than the smallest we ever managed to obtain manually.

\section{Mechanical properties}

The material type 1 has a Young's modulus of $200 \times 10^{8}$ dyn $\mathrm{cm}^{-2}$ and a Poisson's ratio of 0.3. Material type 2 has a much higher stiffness and material type 3 has a much lower stiffness. For a discussion of the basis of these values see Funnell and Laszlo (1982); they are the same as used for our earlier model (Funnell and Laszlo, 1978). The material density (which was not needed in the earlier static model) is taken as $1 \mathrm{~g} \mathrm{~cm}^{-3}$ for materials 1 and 3 . For material 2 it is taken as zero, since the manubrial mass is included in the ossicular load.

The ossicular load is taken to be an angular stiffness of $28 \mathrm{k} \mathrm{dyn} \mathrm{cm}$ (as in Funnell and Laszlo, 1978) and a moment of inertia of $0.2 \mathrm{mg} \mathrm{cm}^{2}$ (as in Funnell, 1980; discussed in detail in Funnell, 1975), both acting about the axis of rotation.

\section{E. The finite-element program}

The finite-element program used here is a modified subset of SAP IV (Bathe et al., 1974). It is essentially the same program as that used in Funnell and Laszlo (1978), with the addition of the feature of "slave" nodes. This feature allows certain nodes to be slaved to a "master" node, so they share in its motions without requiring additional degrees of freedom in the system matrix equation. This feature is used to represent the manubrium as a rigid body with a fixed axis of rotation parallel to the $x$ axis. One node on the axis of rotation has a single degree of freedom corresponding to $\theta_{x}$, that is, to rotation about the $x$ axis. For all nodes on the manubrium, the degrees of freedom corresponding to $y, z$, and $\theta_{x}$ are all slaved to the corresponding degrees of freedom on the axis of rotation; this means that the $y$ and $z$ displacements of the nodes are proportional to their distances from the axis, and their rotations $\theta_{x}$ are equal to that on the axis of rotation. The main advantage of this approach is that it avoids the numerical problems introduced by having some element stiffnesses much larger than others in order to represent the rigidity of the manubrium. When this feature was tested on the 1978 eardrum model it resulted in differences of about $10 \%$ in the displacements calculated, corresponding to a more accurate matrix inversion.

From the above discussion it can be seen that the model being presented here is almost equivalent to the ones described in earlier papers. The most noticeable difference is the correction of an error in the 1978 paper. In the calculations for that paper some of the nodes in the pars tensa were accidentally constrained to zero displacements. This resulted in displacements that were somewhat too small, and a vibration pattern with its anterior peak shifted towards the manubrium. The effects were relatively small, largely because the behavior of the eardrum is dominated by its larger posterior portion. In the following discussion of the effects of varying mesh resolution, the displacements for the corrected 1978 model are shown.

\section{EFFECT OF MESH RESOLUTION ON STATIC BEHAVIOR}

The accuracy of a finite-element model is partly determined by how fine the element mesh is. A coarse mesh may 
introduce large errors due both to the discretization of the underlying partial differential equations, and to the inaccurate representation of the geometry of the object modeled. Unfortunately, the use of fine meshes may incur very large costs. The eardrum model used in earlier papers had quite a coarse mesh due to the restrictions of the computer software being used. It is important to examine the effects of mesh resolution, especially since we wish to consider the higherfrequency behavior of the model where the vibration patterns become more complex and hence more sensitive to limited mesh resolution.

Figure 2 shows the maximal drum displacement and the manubrial-tip displacement, at $100 \mathrm{~dB}$ SPL, calculated for values of the resolution parameter $n$ between 6 and 15. It can be seen that the maximal drum displacement is almost constant at about $700 \mathrm{~nm}$ for $n$ equal to 12 or more, indicating convergence of the behavior of the model. The error in the worst case $(n=7)$ is less than $30 \%$. The pattern is similar for the manubrial-tip displacement. The ratio of the two displacements, which serves as a single quantitative measure of the shape of the vibration pattern, only varies by about $12 \%$ over the whole range of $n$.

The finite-element mesh used in the 1978 paper had a resolution approximately equivalent to $n=8$. The present results confirm, then, that the mesh of the earlier model was not so coarse as to introduce errors that were serious compared to the other sources of error in the model. The displacements calculated with that original mesh are included in Fig. 2: they are roughly $25 \%$ lower than the automatically generated mesh with the same resolution. This discrepancy is presumably due mainly to the small difference in threedimensional shape resulting from the different ways of implementing the curvature as discussed above.

\section{NATURAL FREQUENCIES AND MODE SHAPES}

The undamped natural frequencies of the eardrum model are obtained by calculating the eigenvalues of the system stiffness matrix. The eigenvectors give the vibration pat-

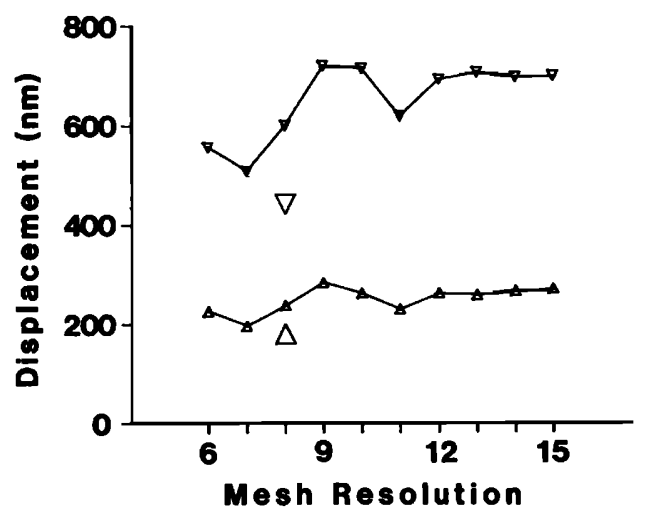

FIG. 2. Convergence of computed displacements as mesh resolution increases. The horizontal axis is the mesh-resolution parameter $n$. The points on the upper curve give the maximal displacements of the pars tensa, while those on the lower curve give the displacements of the tip of the manubrium. The large symbols indicate the corresponding displacements for the 1978 model (Funnell and Laszlo, 1978). terns corresponding to the natural frequencies. For this paper the first six natural frequencies and the corresponding mode shapes have been calculated, using a mesh resolution of $n=12$.

Figure 3 shows the vibration patterns calculated using the standard parameter values given in Secs. IB and ID. The lowest natural frequency is at about $1.8 \mathrm{kHz}$, and the next five natural frequencies all occur within the next $1.4 \mathrm{kHz}$.

To check that the mesh resolution at $n=12$ is adequate, the natural frequencies were also calculated with $n=15$. The differences were all less than $40 \mathrm{~Hz}$ except for a difference of $125 \mathrm{~Hz}$ in the third natural frequency.

Most of the first six natural frequencies correspond to resonant vibratory modes in the posterior region of the eardrum. In Fig. 3, the vibration patterns for the third and fourth natural frequencies both involve a single anterior modal pattern which is combined with two different posterior patterns. In a case like this where two natural frequencies are very close together, the details of the mode shapes are very sensitive to the details of the model: a slight change in the mesh, for example, may cause the anterior mode to appear at a single natural frequency unassociated with any posterior mode. The features that appear consistently here as well as in Funnell and Laszlo (1980) are that the first several natural frequencies occur between about 2 and $3 \mathrm{kHz}$ and correspond to a single anterior resonant mode and several posterior modes of increasing complexity.

The best available experimental data for comparison with these model results are the holographic data of Khanna and Tonndorf (1972). Figures 7 and 9 in their 1972 paper illustrate the progressive breakup of the vibration pattern of the cat eardrum as the frequency is increased. Three things should be borne in mind when comparing their data to the vibration patterns shown here. The first is that their data correspond to arbitrary forcing frequencies, and not to the actual resonances of the eardrum. Second, their data include the internal and external damping of the eardrum, whereas the model here ignores damping. The third point is the considerable variability in the shapes of the experimentally observed vibration patterns as they become more complex. With these points in mind, one would judge that their data

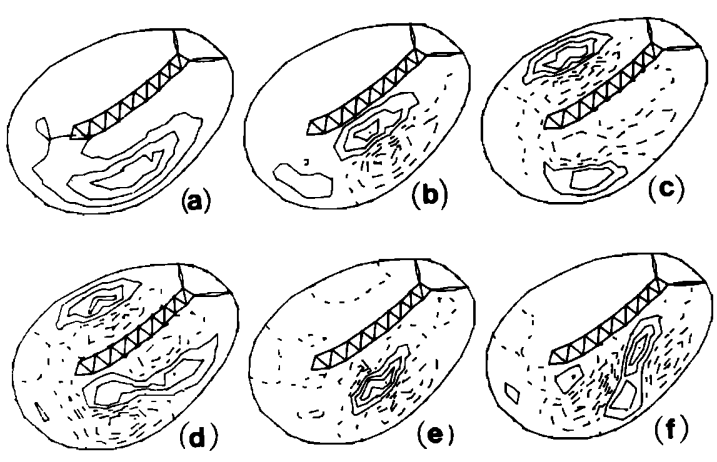

FIG. 3. The vibration patterns corresponding to the first six natural frequencies, for the standard set of parameters. The contours represent lines of constant vibration amplitude. The solid contours represent positive displacements, the long, dashed ones represent negative displacements. The short, dashed lines indicate zero amplitude. (a) $1.761 \mathrm{kHz}$, (b) $2.312 \mathrm{kHz}$, (c) 2.590 $\mathrm{kHz}$, (d) $2.622 \mathrm{kHz}$, (e) $2.926 \mathrm{kHz}$, (f) $3.194 \mathrm{kHz}$. 
suggest that the first natural frequency occurs closer to 3 than to $2 \mathrm{kHz}$.

\section{EFFECTS OF PARAMETER VARIATIONS}

The values of the parameters used in the present model, as in Funnell and Laszlo (1978), are rough estimates based on a variety of arguments, and no attempt has been made to optimize the choice of parameter values in order to fit the holographically observed vibratory behavior. In this section the sensitivity of the model to variations in some of the parameters will be investigated.

Figure 4 shows the effects of varying the two parameters that represent the load on the eardrum due to the ossicular chain and cochlea. In each part of Fig. 4 and of Figs. 5 and 6, the values of the first six natural frequencies are plotted as functions of a particular parameter, with the other parameters having the standard values given above. Solid lines are drawn joining points having similar modal vibration patterns. Dashed lines are used when two vibration patterns are different but apparently related, as when an anterior resonance splits and starts to appear at two different frequencies, for example. No lines are drawn when it is not clear how a modal pattern at one parameter value has evolved from the neighboring value. In some cases, especially when the natural frequencies are close together, there is considerable intermingling of modal patterns as the model parameters vary.

For both ossicular parameters there is a noticeable but relatively small effect on the lowest natural frequency: the frequency increases with increasing stiffness and decreases with increasing inertia, as expected. Doubling the ossicular stiffness increases the lowest natural frequency by about $3 \%-5 \%$; halving the moment of inertia causes an increase of about $6 \%-12 \%$. The ossicular load has very little effect on the higher frequencies. This is reasonable since the manubrial displacement is smaller for the higher modes.

The three parts of Fig. 5 present the effects of varying the stiffness, density, and thickness, respectively, of the pars tensa. As with the ossicular load, the natural frequencies

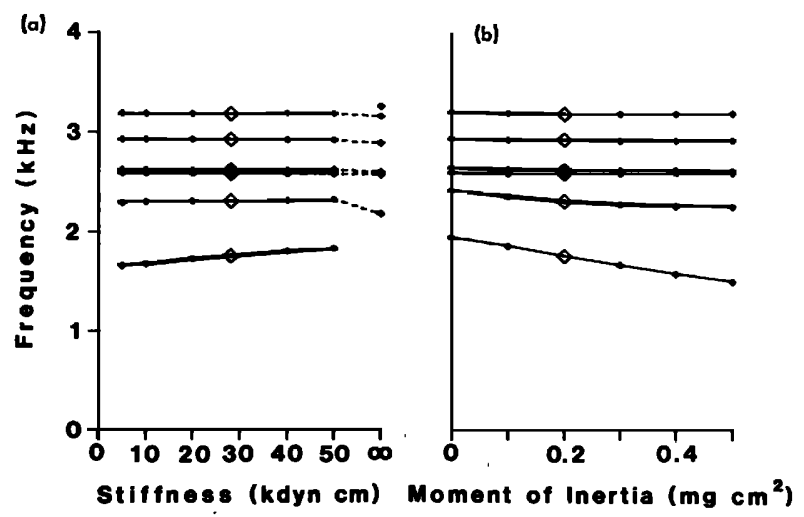

FIG. 4. Effects of varying ossicular parameters. In each part, the six curves represent the first six natural frequencies as functions of a particular parameter, with the other parameters equal to their standard values. The large symbols indicate the standard value of the parameter being varied. (a) Angular stiffness of the ossicular load, (b) ossicular moment of inertia.

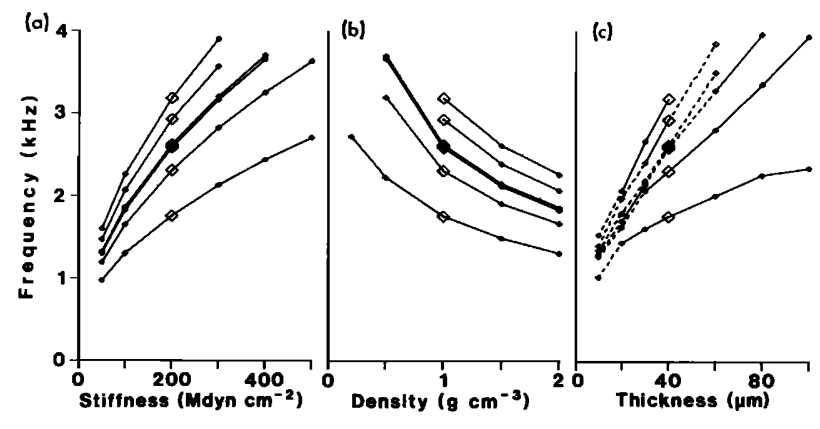

FIG. 5. Effects of varying properties of pars tensa. (a) Stiffness (Young's modulus), (b) mass density, (c) thickness.

increase with increasing stiffness and decrease with increasing mass, but in this case the variations are much greater: doubling the stiffness causes an increase in the lowest natural frequency of about $35 \%-40 \%$, while halving the density causes an increase of about $25 \%-35 \%$. Increasing the thickness increases both the stiffness and the mass, with opposite effects on the natural frequency, but since the stiffness increases more rapidly with thickness than does the mass the overall effect is an increase in natural frequency with increasing thickness: doubling the thickness causes an increase of about $20 \%-30 \%$ of the lowest natural frequency. The effects on the higher natural frequencies are even more marked.

The results of modifying the three-dimensional shape of the model are less straightforward than when one modifies the material properties. Figure 6(a) shows the effects of varying the curvature of the sides of the drum, as represented by the normalized radius of curvature $c$. Increasing $c$ (that is, making the drum's radial fibers flatter) has different effects on the different modes: the two lowest natural frequencies pass through a shallow maximum in the vicinity of $c=1.5$ to 2 , while the higher frequencies decrease more or less monotonically with increasing $c$. A value of $c$ in the neighborhood of 1.4, which subjectively is not an unreasonable estimate for the curvature actually found in the eardrum, is a good compromise, giving a large lowest natural frequency without decreasing the higher ones too much.
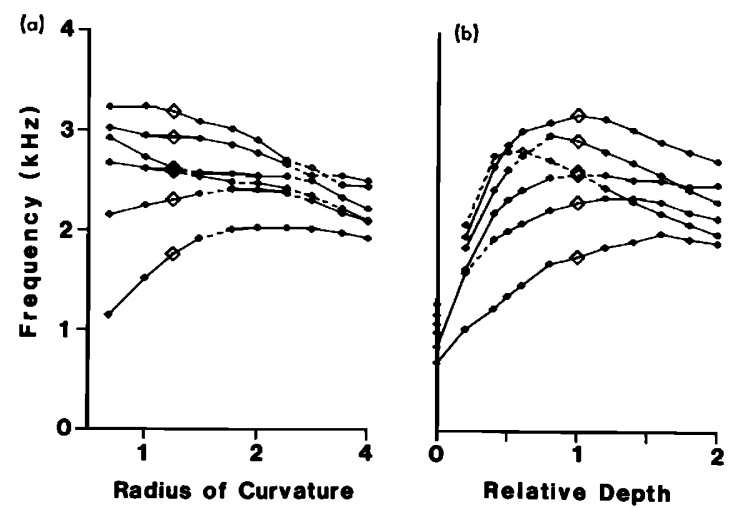

FIG. 6. Effects of varying three-dimensional shape. (a) Normalized radius of curvature, (b) relative depth. 
Figure 6(b) shows the effects of varying the depth of the cone formed by the eardrum. The variation is expressed in terms of a "relative depth," with the standard model shape having a value of one; as in Funnell and Laszlo (1978), the $z$ coordinate of each point is multiplied by this relative depth. Thus a value of zero represents a perfectly flat eardrum, and values greater than one represent eardrums which are exaggeratedly deep. All of the first six natural frequencies increase markedly as the relative depth is increased from zero. The lower ones tend to reach a plateau at about one, while the higher ones all decrease as the depth is increased beyond one. A relative depth in the vicinity of one gives nearly maximal values for all but one of the natural frequencies computed here.

\section{DISCUSSION}

The hierarchical mesh-generation procedure described briefly in this paper greatly facilitates the preparation of finite-element models of irregularly shaped structures like the eardrum. Using the ability to produce meshes of varying resolution, it is possible to select a mesh which provides a reasonable combination of cost and precision.

The natural frequencies and mode shapes calculated with the present model are in reasonable agreement with experimentally observed results, indicating that the parameters of the model are at least of the right order of magnitude. Most of the parameters are based on totally inadequate experimental data. Considerable refinement of the model is possible, not only through better estimates of various parameters but also by relaxing certain oversimplifications in the model. For example, the eardrum is modeled here as being uniform and isotropic although it is neither.

In order to provide some insight into the functional significance of different aspects of the model, the effects of systematically varying several parameters have been described. It is interesting to note that, in the case of both the curvature parameter and the depth parameter, the standard value in the model is in the right neighborhood to broaden the frequency range as much as possible. In our discussion of the low-frequency model (1978) we pointed out the apparent disadvantage of the conical shape of the eardrum in terms of low-frequency sensitivity. The curves in Fig. 6 suggest that the conical shape, and also perhaps the curvature, may serve to extend the frequency range of the eardrum.

It is seen from Fig. 4 that the ossicular parameters have little effect of the natural frequencies and mode shapes of the eardrum. This obviously does not mean that these parameters are not important in determining the transmission characteristics of the middle ear. It is necessary to include the effects of damping in the model so that the displacements, including the ossicular displacement, can be calculated as functions of stimulus frequency.

\section{ACKNOWLEDGMENT}

This work was supported by the Medical Research Council of Canada.

Bathe, K.-J., Wilson, E. L., and Peterson, F. E. (1974). "SAP IV. A structural analysis program for static and dynamic response of linear systems," Rep. No. EERC 73-11, Univ. California, Berkeley.

Crane, H. L., Jr., Gibbs, N. E., Poole, W. G., Jr., and Stockmeyer, P. K. (1976). "Algorithm 508. Matrix bandwidth and profile reduction," ACM Trans. Math. Software 2, 375-377. Complete source in Collected Algorithms from ACM.

Funnell, W. R. J. (1975). “A theoretical study of eardrum vibrations using the finite-element method," Ph.D. thesis, McGill University, Montréal, updated 1976.

Funnell, W. R. J. (1980). "Natural frequencies of a finite-element model of the cat eardrum," J. Acoust. Soc. Am. Suppl. 1 67, S88.

Funnell, W. R. J., and Laszlo, C. A. (1978). "Modeling of the cat eardrum as a thin shell using the finite-element method," J. Acoust. Soc. Am. 63, 1461-1467.

Funnell, W. R. J., and Laszlo, C. A. (1982). "A critical review of experimental observations on ear-drum structure and function," ORL 44, 181-205.

Hazony, Y. (1979). "Algorithms for parallel processing: curve and surface definition with $Q$-splines," Comp. Graph. 4, 165-176.

Khanna, S. M., and Tonndorf, J. (1972). "Tympanic membrane vibrations in cats studied by time-averaged holography," J. Acoust. Soc. Am. 51, 1904-1920. 